\title{
AGRESSION AGAINST MEDICAL DOCTORS - GENESIS AND MANAGEMENT APPROACHES
}

\author{
G. Pavlova*, E. Petrova-Geretto \\ Faculty of Public Health, Medical University-Sofia, Sofia, Bulgaria
}

\begin{abstract}
Aggression in society is not an isolated phenomenon. Violence against medical professionals during their duty is seen more and more frequently.

This work aims to analyze the genesis of the increasing public issue related to aggression against health workers, and to identify possible ways to address aggressive behavior of patients and/or their relatives.

Materials: A review of information on cases of violence against medical professionals for 2014-2017 presented by the Bulgarian Medical Association, the Prosecutor's Office and health establishments was made. A survey among 1409 medical professionals and 1373 patients was conducted.

Results: During the research period, cases of aggression against health professionals were on average of 70-80 cases annually, while the judicial authorities passed only four effective sentences.

Every second health professional interviewed has been a victim of aggression, in most cases verbal. As reasons behind aggressive behavior, patients indicated first low level of culture, lack of values, alcohol and drug use. Most patients do not approve of the healthcare system and identified its imperfections as a reason for rude behavior towards the health facilities' staff. Only $9 \%$ of the respondents were dissatisfied by the doctors' attitude towards patients. Both groups of respondents indicated that legal measures in the Criminal Code were ineffective, failing to act as prevention.

Survey participants suggested unification of efforts of institutions, professional and patient organizations, media, to restore confidence between doctors and patients.

Conclusion: Stopping aggression against health professionals, building up intolerance to this phenomenon, changing the public attitude needs a long and dedicated work demonstrating the social significance and responsibility of medical profession.
\end{abstract}

Key words: aggression against medical professionals, patients' violence against doctors, criminalization of the assault on health professionals.

Aggression in society unfortunately is not an isolated phenomenon. It is encountered ever more frequently in every societal sphereeducation, health care, on the streets, in the family.

Medical professionals can be victims of aggression by patients or their relatives, colleagues or management as result of dissatisfaction, disagreement, competition or mobbing. Various forms of violence represent considerable psycho-social risk at the work place.

The present research focuses on aggressive patients' behaviour against medical professionals during the performance of their

\footnotetext{
*Correspondence to: Dr. Galinka Pavlova, $M D$, and Faculty of Public Health at MU-Sofia, doc.gpavlova@gmail.com
}

duties, the root cause of the issue and prevention efforts.

Occurrence of aggression against medical professionals is not only a national problem. Representatives of 12 European Physicians' Associations met in Ljubljana between the 13th and 16th of September 2017 to discuss this phenomenon, to exchange experience of how to manage it and last, but not least to adopt a Declaration against violence against medical professionals.

According to representatives from Bosnia and Herzegovina violence against physicians exists but it is belittled and remains unannounced. Society treats aggression as isolated case and not as a common, systematic problem. Medical professionals are not legally protected like civil servants such as policemen, judges, ministers etc. The Chairperson of the Medical Association of the Federation of Bosnia and 
Herzegovina has undertaken the initiative to collect data on cases of aggression against medical professionals and amendment to the Penal Code. According to d-r Goran Pavic "violence against physicians is a particular type of violence by patients, their relatives, friends as well as colleagues and officials during the performance of medical duties but also outside of medical establishments. This violence is expression of the perpetrators' wishes, expectations, dissatisfactions, demands for more health rights, demands for inexistent health rights, completion, disagreement, mobbing expressed by shouting, verbal offences, threats, psychological and physical threats, even murder". Physicians are more often reported in media as participants in corruption practices because this news is more interesting rather than physicians as violence victims.

Violence against physicians is a serious issue in Albania which has intensified over the last two decades. On the surface only the tip of the iceberg is visible as not all cases are reported. Aggression both verbal and physical hurts medical professionals, destroys physicianpatient relations, worsens quality of medical services and impacts negatively the whole society. The reason behind this phenomenon, according to Albanian colleagues, is the crises of trust as result of the ineffective healthcare system management exemplified by pharmaceutical and medical consumables deficits. The lack of communication between physicians and patients with regards to diagnostic and therapeutic expectations and low level of societal health culture are also reasons for conflict. Albanian Penal Code defines violence in art. 237 as interference when on duty and art. 238 threat against officials, which are applied in cases of violence against medical professionals.

In Republika Srbska there are two to three cases annually (2015-3, 2016-2, 2017-2). Physician on duty is not an official as policemen, judges and etc. and he/she is not protected against patients' aggression.

A questionnaire among 350 physicians, distributed in 2007 by the Polish Association of physicians and dentists confirmed that $51 \%$ were subjected to violence and interference, $10 \%$ were victims of physical aggression yet only $18 \%$ of the cases were reported to police. In order to manage aggression the Polish association organizes training of medical professionals on the topics of development of skills for avoiding and reducing violent behaviour, improvement of work organization,
PAVLOVA G., et al. provision of legal protection for physicians, development of online monitoring platform for follow-up on aggression and other problematic behaviour against health professionals www.agresja.hipokrates.org - „Monitorowanie agresji w ochronie zdrowia" (MAWOZ)". In 2010 the Polish association of physicians and dentists established the Bureau of Physicians' Rights whose task is to defend this professional group. The main sphere of the Bureau's work is to consult physicians on issues, related to their profession such as legal help, for example, in cases where the good name and/or reputation of a physician is in question because of defamation and slander or if there is physical attack against a physician. The Ombudsman on the rights of physicians defends the dignity of medical profession as well as the collective interest of all physicians. Both the Bureau of Physicians' rights and the Ombudsman on the rights of physicians have analogy on regional level in the Regional Physicians' Associations.

Slovenian representatives point out that the most common violence in the country is psychological violence against family doctors. In addition to verbal and physical aggression, sexual and cyber violence are encounter with increased frequency. In an attempt to find a solution, the legislator has regulated in art. 52 of the Law on patients' rights that "patients must treat health professionals with respect". The zero tolerance approach is further exemplified by the establishment in 2016 of a multidisciplinary working group at the Slovenian physicians' association which meets every two months. Part of the efforts geared towards the violence in healthcare are training of medical staff in communication and deescalation; mounting of an automatic alarm system (red button) in emergency wards and $\mathrm{X}$-ray monitor for patients and visitors.

Precautionary measures in the UK include a register of violence- inclined patients (labelling of violent patients) and distribution of this information to all providers of health care. Violent patients are stripped of their right to home visit by a physician and the physician has the right to strike off the practice's patient register a violent patient. With the aim of prevention, the community is informed regularly of potentially dangerous individuals who reside in the area.

In Austria, according to a survey in 2006- 2007 in eight Stephan Dorfmaster hospitals and the Vienna Hospital Association, reported that the highest percentage of violence happens in psychiatric, geriatric and emergency wards. 
Two thirds that is $75 \%$ of violence is verbal including threats and insults. Less frequent but still a very high percentage of $44 \%$ of physical attacks are reported by respondents. The most common reasons for violence are quoted as follows- $33 \%$ prolonged waiting time and $17 \%$ physicians' decline to fulfil a patient's wish. According to legislation, violence against medical professionals is a criminal offence and physicians have the right to self-defence. The employer is obliged to guarantee the health and safety of employees (Austrian Civil Code, Law on employee safety, Employee Code).

Croatian Physicians' Association study among 1587 physicians concluded that $97 \%$ of respondents report that physical safety and security in health establishments are inappropriate. In January 2017, the Croatian Physicians' Association suggested to the Minister of Justice a number of amendments to the Penal Code, which could guarantee proper protection of physicians and health professionals during delivery of medical help. The Association's website provides the possibility for immediate reporting of aggression and swift provision of legal counsel on behalf of the professional body. The Croatian Physicians' Association organises educational workshops for medical professionals as well as a public campaign "Zero tolerance against violence".

A questionnaire in Romania on the issue of aggression against physicians, respondents identified the following results: $36,8 \%$ reported psychological stress and exhaustion; $15,7 \%$ negative influence on their family and quality of life; $14,6 \%$ depression and anxiety; $13,9 \%$ interruption of professional work; $10,9 \%$ insomnia; 7,6\% worsen personal health; $2,9 \%$ diseases related to post-traumatic stress; $0,9 \%$ changed profession. Since 2006 art. 652 regulates penalty for all types of violence against physicians during their duty. Threatsprison sentence between 6 months to two years or a fine; physical violence or other violent behaviour- prison sentence between 6 months and 3 years; personal injury- prison sentence between 6 months and 6 years; serious personal injury- prison sentence between 3 to 12 years.

According to the Czech Physicians' Chamber, the reasons behind violence against professionals in health care are the negative statements of politicians and in detail media reporting of unsupported by evidence and unproven physicians' mistakes. Increased aggression is one of the reasons why health professionals leave the country which in its turn leads to lower quality of health services and consequently, further negativity towards physicians.

The German medical association found in 2015 via a national survey that $91 \%$ of GPs were victims of patients' aggressive behaviours, majority of medical doctors felt safe in their practices, yet they felt in danger during home visits. One of the reasons behind increased aggression against medical doctors is the excessive workload of health professionals and subsequent longer waiting times.

In order to manage the issue of "violence against medical doctors" in the USA, there are guards/ policemen in emergency units, there are isolation rooms for aggressive patients, waiting and isolation rooms are under video surveillance. The community is informed of these measures as primary prevention against violence against health staff.

During the last two-three years we are witnessing in Bulgaria a rising trend of aggression which has become systematic event.

Bulgarian Medical Association has exercised pressure in order to criminalise aggressive behaviour and amendments were made in the Penal Code. Article 116, paragraph 2 heavily punishable crime for murdering medical specialist and article 131, paragraph 2 for personal injury. The despicable physical attack against d-r Albena Gatova, M.D. on the $6^{\text {th }}$ of July 2017 by a father of a new-born baby caused public outcry. This crime motivated the Bulgarian Medical Association to collect data and undertake research on the cases of aggression/violence against medical professionals during their duty. Summarizing information from Ministry of Interior, Prosecutors office and health establishments for the period between 2014 to mid-2017, it was found that on average there are between 70 to 80 cases of violence against physicians, 100 are there registered attacks against emergency/ ambulance medical teams and there are only 4 effective sentences.

In order to discover the root causes of violence, the critical issues as well to study the public opinion and implement measures against violence in health establishments, the Bulgarian Medical Union undertook questionnaire survey among health professionals and patients. There were 2782 respondents, 1409 were medical professionals and 1373 were patients. The questionnaire survey took place between the $20^{\text {th }}$ of July and 
$10^{\text {th }}$ of September 2017. Answers were received online and on paper.

Most often aggression is found in emergency/ ambulances and in emergency units in health establishment- $90 \%$. Least aggression is to be found in GP practices- $3.1 \%$ most likely due to the fact that GPs know very well their patients and could predict patients' behaviour. Almost every second respondent or $42 \%$ has been victim of aggression; $36 \%$ of them more than three times while only $16 \%$ were subjected to
PAVLOVA G., et al. aggression only once. In most cases aggression was verbal- $81 \%, 1 / 5$ of respondents did not undergo forensic medical expertise as aggression was predominantly verbal. $3.6 \%$ respondents suffered personal injury, which was also left without consequences.

Considerable part of patients reported dissatisfaction from health services in Bulgaria. Only 5\% approve of the health system (Figure 1).

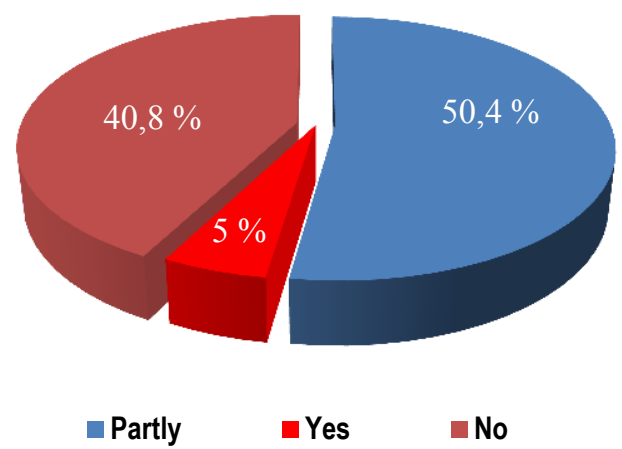

Figure 1. Are you satisfied with health services in Bulgaria?

Dissatisfaction from the system is easily transferred on medical doctors and escalated in aggressive patient behaviour. The most serious issue which causes dissatisfaction is out-of pocket payments (Figure 2).

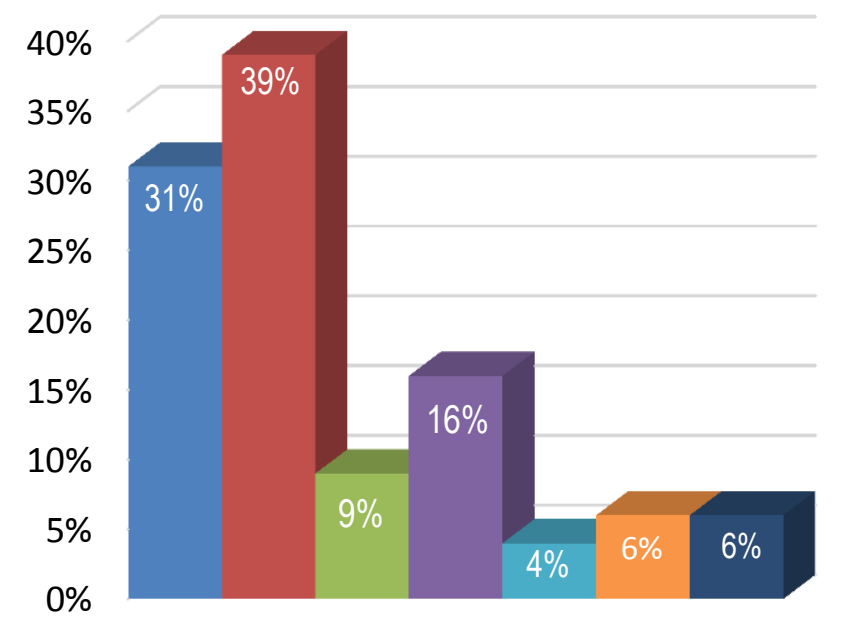

Figure 2. What are you dissatisfied with? / open question/
Referral limits

- Co-payment

- Lack of commitment and rude behaviour on behalf of physicians

Ineffective healthcare System organization, chaos
Similarly, the World Bank reports that $48 \%$ of households make out- of-pocket payments. Additional expenses are frequently due to a number of limitations such as hospital limits and regulatory standards in outpatient care. Restrictive limits restrain access to the system while on the other hand they contribute to over-hospitalization and unreliable statistics. Dissatisfaction is directed predominantly against the system- lack of organization, sizable bureaucracy and only 9\% are dissatisfied by physicians' attitude.

Patients' answers diverge from those of medical doctors. When patients were asked if they manifested aggression against a health professional, $5 \%$ answered affirmatively, while 95\% denied. $6 \%$ admitted physical aggression against physicians. 
Half of both groups confirmed that they are informed that attack against medical professionals is a criminal offence. Physicians and patients alike deem unnecessary to amend legislation that is both groups belive that legal provisions are sufficient but not properly applied.

Both groups of respondents reported that aggression is a result of provocative behaviour
PAVLOVA G., et al. on behalf of physicians: $7.7 \%$ of medical doctors and $14.8 \%$ of patients. That is twice as many patients believe that physicians behaviour is what causes aggression. The high percentages on the diagram indicate low personal culture (lack of a value system), drug and alcohol use, system's imperfections.

(Figure 3)

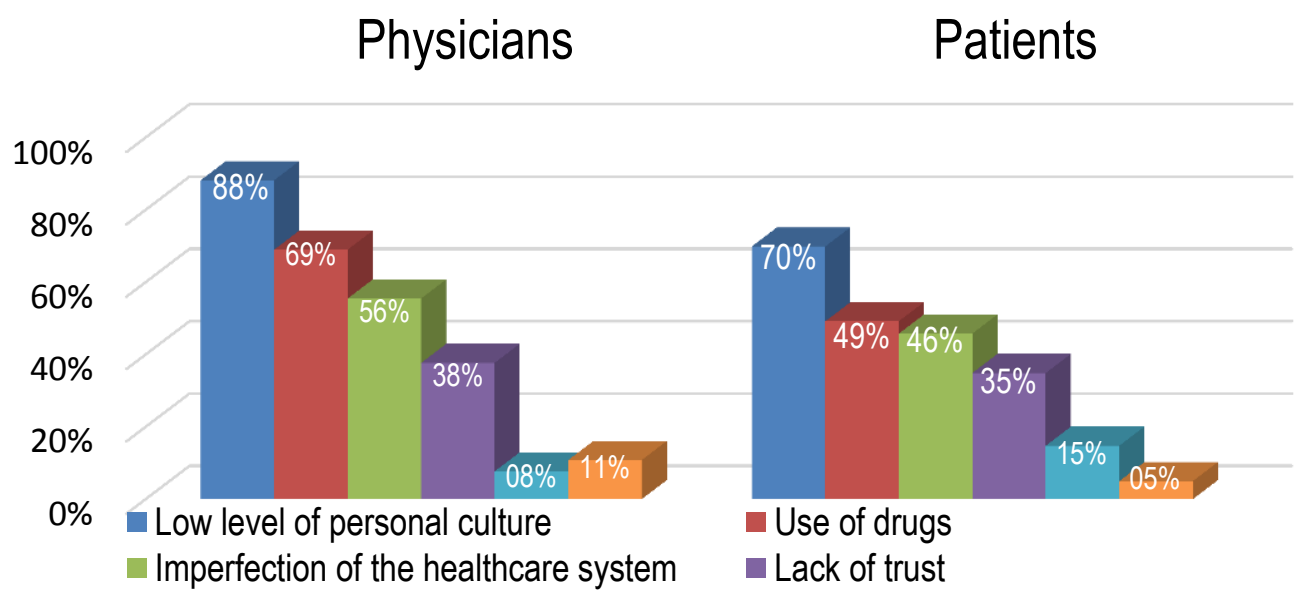

Figure 3. What is the genesis behind agression against medical professionals? /more than one answer is possible/

According to health professionals severe sanctions and effective sentences are needed in addition to tighter security and community work. Yet, $30 \%$ of medical respondents critically assessed the need of improved communication skills which would bring about a subsequent change in societal attitudes (Figure 4).

\section{Physicians}

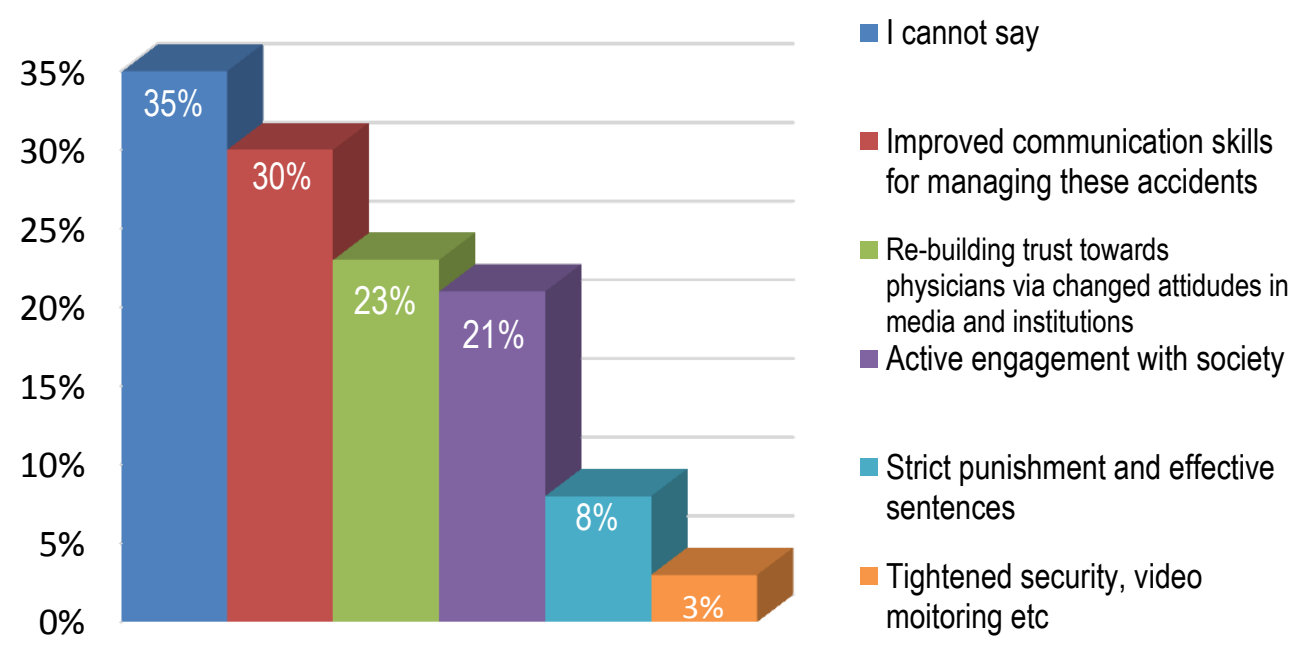

Figure 4. What preventive measures should be undertaken in order to put a stop on agression? /open question /

To the same question patients indicated in similar percentages preventive measures such improved communication skills. Patients insist on proper application of the law and 22\% demand stricter punishment. 
The questionnaire's results and data from health establishment on aggression over the past three years can be summarized as follows:

- Most often aggression against health professionals takes place in emergency care/ ambulance services and emergency wards.

- Patients' dissatisfaction with system failures are transferred to physicians.

- The most common reasons behind aggressive behaviour of patients and/or there relative are low personal culture and morality, drug and alcohol use, negative institutional and media representation of the medical profession.

- Legal provisions are not applied effectively and cannot serve as prevention to violence.

- Insufficiently developed ethical communication between physicians and patients.

- Impaired patients- physicians trust.

Building on experience of other countries in prevention measures against aggression, Bulgarian Medical Union proposes to undertake institutional and social measures, including:

1. Systematic and strict application of legal provisions for aggression as a serious criminal offence.

2. Development at the Ministry of Interior of a unified system for receiving signals for aggression against health professionals

3. Development of a monitoring system at the Bulgarian Medical Union for root cause analysis and adoption of corrective actions.

4. Sharing of information with health establishments for patients- recidivists in order to adopt preventive measure for risk minimisation.

5. When there is a request for home visit for a violence -inclined patient, the visit should be made in the presence of an Interior Ministry representative.

6. Consistent, all-encompassing campaign for changing societal attitudes and for informing society how significant and important is the medical profession in society

7. Improved communication skills of health professionals.

8. Improved information on the rights and obligations of patients.

\section{CONCLUSION}

Aggression is behaviour which is deeply rooted in social and public life. The reasons behind aggression against medical professionals is closely linked to the unresolved issues in the healthcare system.
PAVLOVA G., et al.

Managing of this negative phenomenon is only possible if there are:

- Improved organisation and effective functioning of the system;

- Improved quality of medical services;

- Public recognition of the significance of medical profession.

Practice demonstrates that in order to limit aggression protests via public media are not enough. It is necessary to unite efforts of institutions, professional organisation and media for preventive and positive campaign. Effective management of this negative phenomenon require continuous daily work in order to change societal attitudes and return the trust in the medical profession. By demonstrating to society the excellent examples of dedication, hard work for achieving high level of professionalism and the significance of medical profession, the trust between patients and doctors will be reestablished which will limit aggression against physicians.

In this respect, the Bulgarian Medical Union initiated in September 2017 National campaign against medical professionals under the title "To save a life is dedication and does not deserve aggression" \#OvercomeAnger.

\section{REFERENCES}

1. Tomev, A., Daskalova N., Ivanova V., Violence at the work place in health care in Bulgaria. 2001. Sofia

2. Stoykova, Ts., Aggression as social phenomenon. Articles and research 2013

3. European Convention on Human Rights and Fundamental Freedoms

4. Zografova, Y., Information and processional models- new alternative for studying aggression. Psychological research.2005. book 2

5. Melibruda, Y., Me- you- us. Capacity of psychology for improving communication between people. Sofia. 2017.

6. Anderson, C. and Bushman, B. Human Aggression. Annual Review of Psychology, 53, 27-51, 2002.

7. Bandura, A., 1973. Aggression: A social learning analysis. Englewood Cliffs, NJ: Prentice Hall.

8. http://www.hervoice.com/work.html 